\title{
Synthesizing Verdant Landscapes using Volumetric Textures
}

Fabrice Neyret

\section{$N^{\circ} \mathbf{2 8 4 6}$}

Mars 1996

THÈME 3 



\title{
RINRIA
}

\section{Synthesizing Verdant Landscapes using Volumetric Textures}

\author{
Fabrice Neyret ${ }^{*}$ \\ Thème 3 - Interaction homme-machine, \\ images, données, connaissances \\ Projet Syntim \\ Rapport de recherche $\mathrm{n}^{\circ} 2846$ - Mars $1996-12$ pages
}

\begin{abstract}
Volumetric textures are able to represent complex repetitive data such as foliage, fur and forests by storing one sample of geometry in a volumetric texel to be mapped onto a surface. This volume consists in samples of densities and reflectances stored in voxels. The texel can be prefiltered similarly to the mip-mapping algorithm, giving an efficient rendering in ray-tracing with low aliasing, using a single ray per pixel.

Our general purpose is to extend the volumetric texture method in order to provide a convenient and efficient tool for modeling, animating and rendering highly complex scenes in ray-tracing. We illustrate our method with verdant landscapes such as forests and lawns.

In our previous work, we have dealt with the multiscale volume representation and texel animation. In this paper, we show how to convert usual 3D models into texels, and how to render texels mapped onto any mesh type. Solving these two issues makes the method usable for a designer.
\end{abstract}

Key-words: volumetric textures, complex scenes, mapping, multiscaling, landscapes.

*Fabrice.Neyret@inria.fr http://www-rocq.inria.fr/syntim/research/neyret 


\section{Synthétiser des paysages forestiers à l'aide de textures volumiques}

Résumé : Les textures volumiques permettent de représenter les objets complexes répétitifs comme le feuillage, la fourrure ou la forêt en stockant un échantillon de la géométrie dans un texel volumique destiné à être mappé sur une surface. Ce volume contient des valeurs de densité et de réflectance échantillonnées en voxels. On préfiltre Le texel comme le fait l'algorithme de mip-mapping, ce qui conduit à un calcul de rendu efficace en lancer de rayon avec peu d'aliasing, en utilisant un seul rayon par pixel.

Notre motivation est d'étendre la méthode des textures volumiques afin de proposer un outil efficace et commode pour modeler, animer et rendre les scènes très chargées en lancer de rayon. Nous illustrons notre méthode avec des paysages de végétation comme la forêt ou la prairie.

Dans nos travaux précédants, nous avons traité le problème de la représentation volumique multi-échelle et de l'animation des texels. Dans ce papier, nous montrons comment convertir les représentations 3D usuelles en texels, et comment calculer le rendu des texels mappés sur des maillages quelconques. Résoudre ces deux points rend la méthode réellement utilisable pour un utilisateur.

Mots-clé : textures volumiques, scènes complexes, plaquage de texture, multi-échelle, paysages. 


\section{Introduction}

Geometric complexity is an important aspect of realism in synthetic scenes. This is particularly true for natural scenes such as forests, lawns, fur, etc. In order to represent and render scenes, one has to choose between using a general synthesis process (consisting of interactively modeling meshes, specifying the animation, and launching a renderer), and using a data-specific tool for either the modeling stage or eventually the whole synthesis process. In this paper, we show that for complex scenes, volumetric textures are a good trade off between generality and efficiency.

\section{Classical synthesis process}

It is convenient to use such general tools and representations, since many products are available and existing data can be re-used. The high geometric complexity makes this theoretical solution usually impractical in each of its stages :

- modeling requires a lot of detailed knowledge from the user, and is very repetitive (e.g. each leaf of a tree).

- the generated database is highly memory-consuming. - similarly, the animation needs the user to specify the motion of many components.

- the rendering has to deal with billions of facets, which is costly in time and results in a lot of aliasing.

Although clever optimisations and database structurations exist [FvDFH90], antialiased ray-tracing of such scenes is still very slow. An important aspect lies in the determination of levels of details, which is currently an active research topic. Scanline-renderers with shadow capabilities can also be used, but one has to deal with aliasing, and detailed shadows can hardly be obtained with a wide scene containing fine details.

\section{Specific approaches}

These approaches solve the problem for particular kinds of scenes, either by procedurally describing the objects, or by providing a specific representation and the mean to render it. The first category concerns L-systems, fractals, botanical and physical models [Smi84, PLH88, $\left.\mathrm{dREF}^{+} 88, \mathrm{FR} 86\right]$ which generate polygons. The second category mostly concerns particle systems and some landscape-specialized models [Ree83, RB85, WP95], which use specific representations and rendering techniques. Procedural geometric models do not address the rendering aspect of the problem, and specific representations forbid the use of general modeling tools and existing databases. The integration of different kinds of objects in a single scene can also be limited.
Particle systems, although effective in producing certain effects, have some limitations as a geometric representation :

- the geometric primitive is a one-pixel thick line tracing a trajectory, thus limiting the representable shapes to a narrow family.

- despite the limited individual drawing cost, filtering millions of blades at a far-off distance in the scene is costly.

- the rendering is ad-hoc (painting a color more than computing the local illumination), and can hardly be mixed with a classical scene. For instance, specific extensions have to be introduced in order to deal with shadows.

\section{Volumetric textures}

In our previous work, we have shown how to make volumetric textures an efficient way of representing and rendering complex repetitive data: the precomputed multiscale representation provides the right look of the data at the right scale. The textural approach is limited to scenes which contain some repetitiveness. However it allows us to separate the scales of specification into a local $3 \mathrm{D}$ aspect (the texel pattern) and a wide aspect (the surface on which texels are mapped, and the deformation of these texels). Therefore, volumetric textures provide a relatively general representation of complex scenes, and enable an efficient unaliased ray-traced rendering (see section 2). Thus, volumetric textures are a good trade off between generality and efficiency.

But the texel representation described in previous work is mostly specific, as the texel content has to be specified 'manually' by the user, and the surface has to be discretized by bilinear patches in such a way that one texel corresponds to one patch.

In order to make the volumetric texture model usable, we describe in this paper:

- A way to convert usual object descriptions into volumetric textures (section 3 ), thus allowing the use of existing modeling tools and databases;

- A method of rendering texels mapped on any mesh type (section 4), using texture coordinates such as those for usual 2D textures.

Having solved these two major issues, we illustrate the model by synthesizing verdant landscapes (section 5) using an existing representation of trees and a free mapping on surfaces. 


\section{$2 \quad$ Volumetric Textures}

Kajiya and Kay first introduced texels in 1989 [KK89] mainly to render fur. Despite the fact that the paper contained the basic ideas, a lot of coding is still required to simulate other materials, and rendering is very slow since no multiscale scheme is proposed. Moreover, the surface has to be subdivided into bilinear patches so that one texel corresponds to one patch. However, the look of the resulting teddy-bear is simply marvelous. The authors use a full volumetric storage as a 3D texture pattern (the reference volume stored once) to encode the geometric data, and an underlying surface meshed with bilinear patches (see figure 1). Each texel is mapped exactly upon a bilinear patch and deformed in order to stick to the neighboring texels, thus forming a thick layer upon the surface. The vertical edges (common with adjacent texels) can be 'combed' by the user.

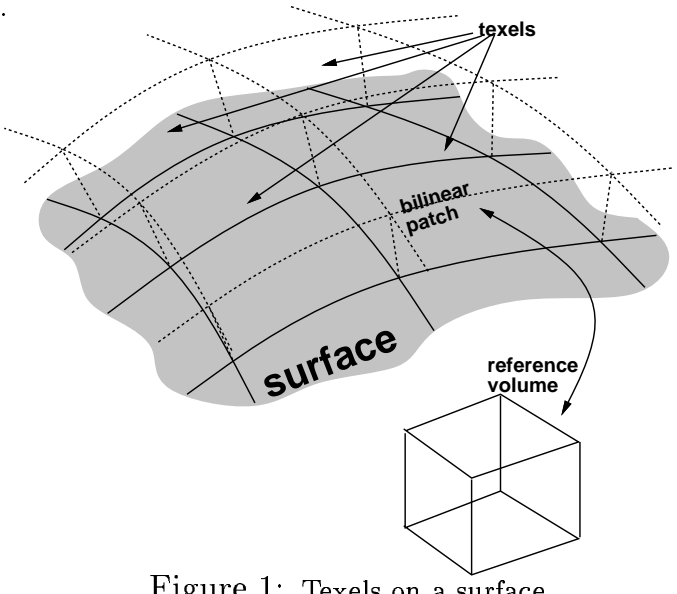

Figure 1: Texels on a surface

Data storage and rendering looks like volumetric rendering, except that the voxel content is not really a density but rather an non-directional probability of occlusion. Another crucial piece of data is added to the voxels: the local photometric behavior, consisting of a local frame and a reflection model, which makes the volume reflect light just like a real object would. The fur rendering implementation models the reflection from a cylinder, and the frame is limited to the cylinder axis. These parameters are not really stored in the voxels since they are constant in the volume: the volume only contains fur, and all the cylinders are parallel (the variation in the combing is obtained at the mapping level).

Thus the volumetric texture is defined by a reference volume, an underlying surface made of bilinear patches, and a thickness vector at each vertex of the surface.

Shinya proposed some improvements in 1992 [Shi92], by storing the occlusion for each spatial direction in order to make the occlusion direction-dependent, and by traversing the volume by steps in each spatial direction. He listed unsolved problems (texel construction from polygonal description, voxels prefiltering), recommended the use of a hierarchical approach, and suggested the use of correlation between voxel contents.

In a previous paper, we proposed an extension to the texel representation [Ney95b], for generality and efficiency: various data can be represented without extra coding, and ray-tracing is achieved efficiently with low aliasing, using a single ray per pixel. Efficiency is obtained with a multiscale scheme similar to the mip-mapping. The key idea lies in the encoding of the local geometry included in a voxel volume, characterized by a Normal Distribution Function (NDF), in such a way that it can be filtered to provide a lower resolution. Thus the volume can be precomputed at each resolution at modeling time, as in mip-mapping. The generality is obtained by keeping enough degrees of freedom in the NDF encoding. We have chosen to approximate this NDF by finding the 'closest' ellipsoid, which needs few parameters and permits various and continuous local shapes, including cylinders and planes.

This ellipsoid plays the role of the reflectance model and the local frame of Kajiya's model. It is determined at modeling time while discretizing a shape into the reference volume. Local illumination is computed at rendering time by numerically integrating the Phong model on the NDF. As for 2D mip-mapping, the two scales closest to the ray thickness are used and interpolated, thus providing filtered data with only one ray per pixel. Therefore, the features of this representation are a probability of occlusion, six ellipsoid parameters, and a pointer to the eight sons (if any), stored in each voxel of an octree.

This solves the filtering problem of volumetric texture. We have demonstrated in another paper [Ney95a] how to animate the representation in the same spirit, separating the scales of control: a pattern can be chosen in a discrete set in a cartoon-like approach, in order to simulate local oscillations or flickering (e.g. moving leaves). The height vectors of the texels can be animated by various ways (e.g. to simulate the effects of wind on a lawn), and the underlying surface can be distorted (e.g. clothes).

Two important unsolved problems were texel construction and texel mapping. Noma [Nom95] proposed a solution to the problem of constructing texels from a particular kind of geometry: sparse little facets (given by the AMAP tree modeling software).

In the next section, we present methods to convert most of the classical geometric representations $\left(\mathrm{CSG}^{1}\right.$, polygonal meshes, L-systems, particle systems, hypertextures,...) into volumetric textures. In section 4 , we deal with texel mapping.

\footnotetext{
${ }^{1}$ Constructive Solid Geometry
} 


\section{$3 \quad$ Texel Building}

Many representations and modeling tools still exist to specify shapes, either general or specific, that users know well and like to use. A convenient way to specify the reference volume content is to convert existing representations into volumes. 3D scanconversion still exists to convert some representations into volumes, but this method parses the whole volume systematically, and is quite costly. Moreover, not only do we need to know the voxel occupation, but also the local reflectance, which implies that the method has to be extended.

CSG, polygonal meshes, implicit functions, particle systems and L-systems [FvDFH90, Smi84, Ree83, RB85, PLH88] are primitive-based techniques: these constructions design a shape by combining simple shapes (the primitives being respectively a solid, a facet, a skeleton element, a trajectory segment, a terminal symbol). The simplicity of these primitives allows more efficient and accurate conversion than straight $3 \mathrm{D}$ scan-conversion: such a primitive can be defined by a distance function, or more conveniently by the distance to its surface, made negative if the tested point is inside the shape. The surface thus corresponds to the zero value of the function, and the volume inside corresponds to negative values. The normals are given by the gradient of the distance function. The conversion of these models is described in section 3.1.

On the contrary, scanner data and hypertextures [PH89] correspond to full volumetric representations, for which data is given at each space location. Local density orientation and octree compression are then determined by scanning the volume. We detail these models in section 3.2 .

We use the texel representation presented in our previous work [Ney95b], in which the data is stored in an octree. To sum up the process, the construction corresponds to the thinnest scale; then a propagation is achieved in the upper stages of the octree in order to precompute the filtered data at all resolutions; finally a compression pass is achieved to suppress unvarying information.

Note that by using a volumetric storage, building a shape looks like painting it into a $3 \mathrm{D}$ drawing. This allows many operations while designing a shape: the drawing function that usually just sets the value into a voxel can be modified to provide some drawing modes. Some useful drawing modes are: adding the value to the previous contents (which can be considered as making the union of shapes), subtracting, multiplying, setting the NDF and not the occlusion probability (which can be compared to bump-mapping on surface), or vice-versa, thresholding the previous value according to the new one, and so on. This is used to implement CSG operators as we will see hereafter.

$\mathrm{RR} \mathrm{n}^{\circ} 2846$

\subsection{Primitive-based models}

Multiscale data structures like octrees are well-adapted to recursive construction [Sam90] : recursive construction supposes us to be able to determine if a given space area is inside, outside or on the frontier of a shape, in the same spirit of the Warnock algorithm in $2 \mathrm{D}$ [FvDFH90]. The function which gives the distance from a tested point to the surface of the shape to be built, conveniently made negative inside the shape, provides a way to determine the status of a voxel regarding the shape (see figure 2):

- if the distance from the center of the current voxel (initially the root of the octree) is greater than the radius of the sphere bounding the voxel, the voxel is outside the shape.

- if this distance is negative, its absolute value being greater than the radius, the voxel is inside the shape.

- otherwise the voxel is probably on the frontier, so we split the voxel into eight sons and apply this algorithm to each son. If the minimum size is reached, we evaluate and store the geometric information corresponding to this area of space.

As explained in [Ney95b], the geometric information consists in an occlusion probability (having the same role as the alpha plane in compositing, see [FvDFH90]) and six ellipsoid parameters approximating the local NDF (the ellipsoid being the support of these normals, and not the distribution. shape.itself).

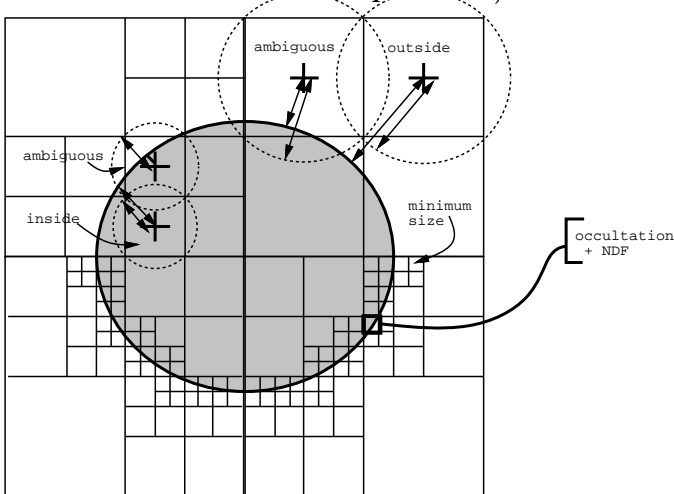

Figure 2: Recursive volumetric construction of a shape (figured in $2 \mathrm{D}$ )

Note that getting the distance function is easy for simple primitives such as sphere or cylinder, and that normals can directly be obtained by computing the gradient.

We have implemented a large amount of primitives ${ }^{2}$, that can be directly used in a declarative script. For instance, a sphere $\{C, r\}$ is obtained with the distance function $d(M$, sphere $)=|\overrightarrow{M C}|-r$, which gives the criterion function $\frac{1}{2}\left(1-\frac{d}{r_{v o x e l}}\right)$ that is bigger than 1 if the voxel is outside the shape, less than 0 if the voxel is inside, and in $[0,1]$ if the voxel contains the frontier.

\footnotetext{
${ }^{2}$ line with cylindrical section, line with rectangular section, plane, half space, sphere, ellipsoid, parallelepiped, cylinder, truncated cone, and some special primitives such as V-grass segment.
} 
CSG operators like union or intersection are easy to implement: a new shape is combined with the previous ones by using a special drawing mode which adds values to existing ones in the voxels instead of setting the new value. Evaluating a whole CSG tree requires computing each sub-tree in separated volumes (which can be memory-consuming), so that each CSG operator only operates either between a drawn volume and a primitive, or between two drawn volumes.

Primitives are also used to paint 'skeleton' representations such as L-systems and particle systems in the reference volume: successive segments are drawn in the volume using cone or cylinder primitives.

Triangular meshes are represented in the same way using the triangle primitive: the Euclidean distance from a point to a triangle can be easily obtained. The $\mathrm{NDF}$ is constant and is equal to the triangle normal. This primitive may seem a bit special in that it is $2 \mathrm{D}$ : we draw the mesh surface and not the enclosed volume (because meshes can sometimes be non-closed surfaces). In fact, the recursive drawing algorithm splits the bounding voxels and stops at the volume resolution, so that there is always a residual thickness.

We have also implemented implicit functions in the same spirit: a distance function can be approximated using the potential, since the only necessary properties for the function are that it be zero on the surface and to not increase any faster than the Euclidean distance (otherwise a voxel can be declared outside instead of ambiguous). Our approximation of the distance is $\frac{1}{\sqrt{p o t}}-1$, where $\operatorname{pot}(M)=\sum \pi_{i} / d_{i}^{2}$, with $\pi_{i}$ and $d_{i}(M)$ being respectively the weight and the Euclidean distance of a skeleton element (point, segment or triangular face).
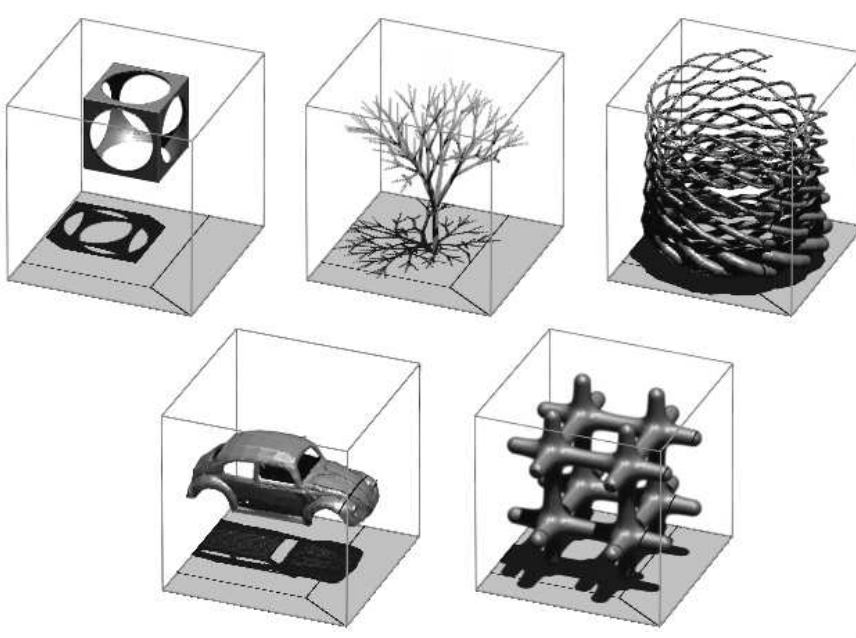

Figure 3: From left to right and top to bottom, texel conversion of: CSG, L-system, particle system, mesh, implicit surface.

\subsection{Volumetric models}

Volumes are another kind of data: medical CT-scan data (explicit field) or hypertexture obtained from
Perlin noise [Per85] (procedural field). Distances cannot easily be obtained since the data does not really represent a surface. We have to use a volumetric scan-conversion: we scan the data at the thinnest resolution, and we create the octree voxel hierarchy "on the fly" only where non empty space is found.

With Perlin noise, the normals are obtained by gradient computation. For pure volumetric density data such as CT-scan data, the density gradient has to be numerically estimated from a neighborhood. This gives poorer results, which shows that direct knowledge of the NDF is more important at this scale than the density distribution.
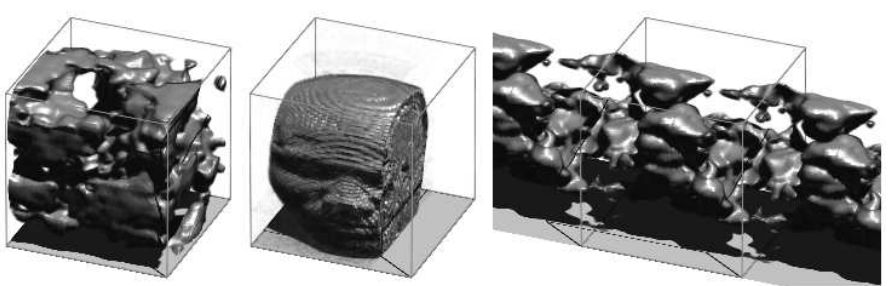

Figure 4: Texel conversion of hypertexture (left) and tomographic image (middle), right: cyclic hypertexture pattern.

\subsection{Discussion of the model}

- Voxelizing a shape brings of course some approximations: from a short range, shapes seem made of bricks and are a bit blurred. To avoid such effects, the volume resolution has to fit the closest point of view requirements. This can be memory-consuming. But one has to keep in mind that texels are built to represent small details in complex scenes; they are not supposed to handle short-range viewpoints. In such cases, a transition with polyhedral geometry can be made [Nom95]. - If duplicated without any variation, the texture will look very repetitive. This problem is dealt with in section 4.3.

- Color representation is not handled by the volumetric model which is a pure geometric representation (it is not easy to filter colored geometry). In previous papers [KK89, Shi92, Ney95b, Nom95], a color material is associated with a whole pattern. We address this problem in two ways: by merging separated texels associated to different materials, which is limited but easy to implement in volume rendering, and by implementing classical textures (picture or procedural) that are used inside texels.
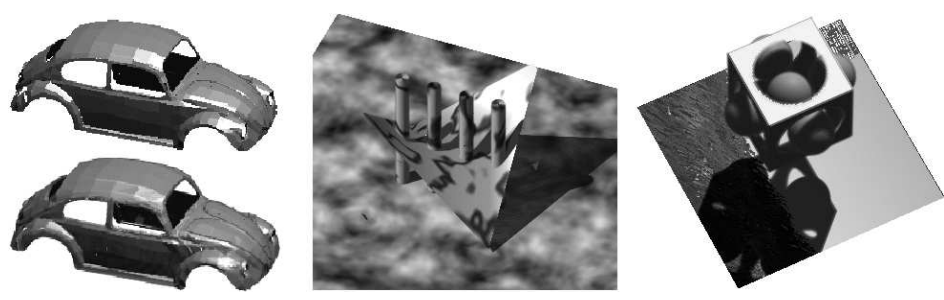

Figure 5: left: real and texelized meshes. middle: textured texel. right: merging of two colored texels. 


\section{Mapping Texels}

Previous publications on texels use a very simple mapping, requiring an underlying surface made of bilinear patches on which texels are placed. The base of each texel is fitted to the patch and their vertical sides are deformed in order to stick to adjacent texels. This constrains the surface mesh, and supposes that the orientation and the size of the texels correspond to the ones of the patches.

We specify in section 4.1 a general texel mapping in the same way that the $2 \mathrm{D}$ mapping is defined, offering new degrees of freedom. The idea is to introduce $3 \mathrm{D}$ texture coordinates inside the volumetric layer to specify the texel mapping. Note that unlike 2D textures where the color computation is separated from the ray scene traversal, in volumes rays have to traverse the texture. We describe this ray traversal in section 4.2. We explain in section 4.3 how to decrease the repetitive appearance of the mapping.

\subsection{Mapping specification}

We use surfaces consisting of triangles and bilinear patches. This allows the use of almost any mesh type, since polygons with more points can be decomposed into triangles and quadrilaterals. The size and the orientation of the polygons are independent of their texel counterparts.

We associate to each vertex a vector $\vec{H}$ called a height vector which controls the texel 'combing', and a vector $\vec{u}=(u, v, w)$ which gives the texture coordinate at the point. Also we define a scalar $d w$ such that the vertex at the top of $\vec{H}$ has a texture coordinate $(u, v, w+d w)$. In most cases $w=0$ and $d w=1$. Kajiya's mapping corresponds to surfaces composed only of bilinear patches, and successive integer values for $u$ and $v$ at vertices (the texel bounds coincide to the patch bounds). Note that we use three different coordinate systems: the spatial one $\mathcal{S}$ where the coordinates are $(x, y, z)$, the thick skin space $\mathcal{F}$ attached to the faces where the coordinates are $(U, V, W)(W=0$ on the face, $W=1$ on the top of the layer, and $(U, V)$ are barycentric coordinates of the face), and the texture space $\mathcal{T}$ where the coordinates are $(u, v, w)$ (see figure 6).

Let us call the thick skin area corresponding to the faces boxes (in Kajiya's mapping boxes and texels are equivalent, i.e. $\mathcal{T}=\mathcal{F}$ ). Assuming that a triangle is a degenerated bilinear patch, the transformations $\mathcal{F} \rightarrow \mathcal{S}$ and $\mathcal{F} \rightarrow \mathcal{T}$ are trilinear interpolations (spatial coordinates and texture coordinates at any point are obtained from the values at the eight vertices).

As in previous work [KK89], a material pointer is associated to each face, which indicates the reference volume to use at this place, and we use classical Phong material description, such as ambiant diffuse and specular color, and roughness. The material specifica-
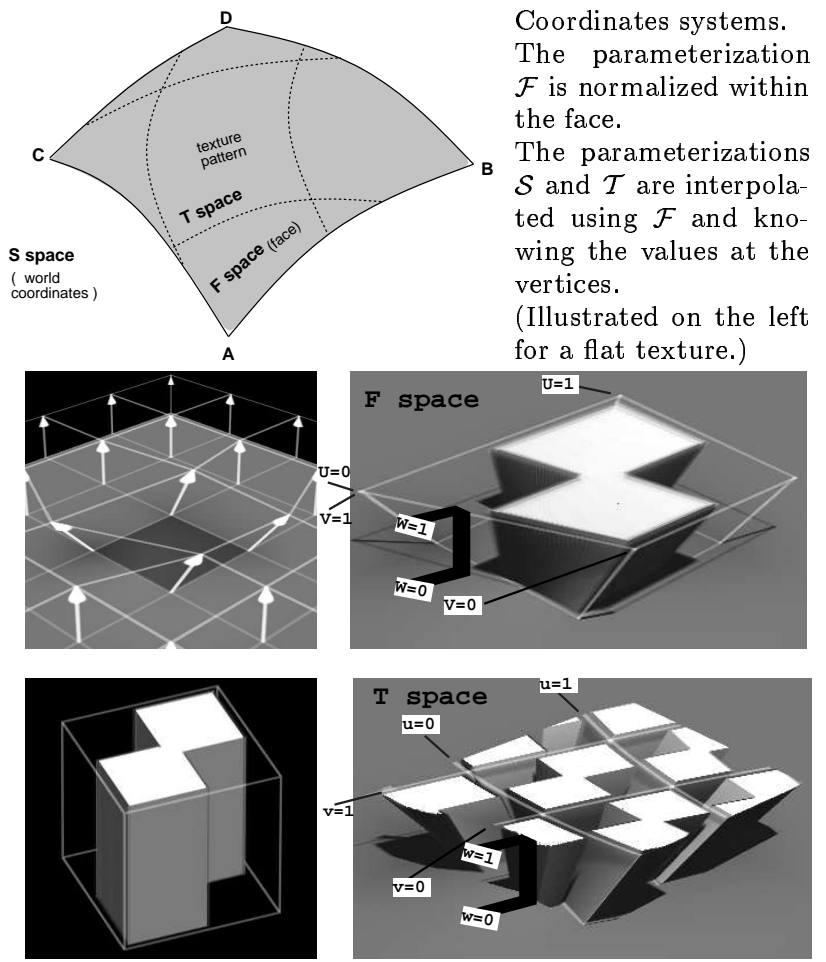

Figure 6: top left: the boxes corresponding to the faces. bottom left: the reference volume. top right: Kajiya's mapping: one texel fits one face (for clarity we have only mapped the central box). bottom right: general mapping defined by the $(u, v, w)$ at the eight vertices (central box only).

tion is slightly more complicated in our implementation since we allow the merging of texels in the same volume, the superposing of texel layers, or the achievement of operations such as scaling, permuting orientation, and so on. Moreover, Phong parameters can be defined by $2 \mathrm{D}$ texture functions such as image mapping or Perlin 3D noise. This is achieved by adding some items and linking the material descriptors used for the same face.

\subsection{Texture traversal while rendering}

We precompute a bounding sphere and a grid around the surface, and a bounding sphere for each face including the texel thickness (see figure 7). During the tracing of a ray, this leads efficiently to the first face intersected by the ray. Note that in our case we have to test the six bilinear faces of the box. Texels stick to each other so that in most cases only the upper and lower surfaces have to be tested for intersection.

When entering inside the skin space, we leave classical ray-tracing. The ray traversal inside this space uses the neighborhood coherence of faces, so that we just have to compute the point where the ray leaves the box, thus providing the next crossed box. This part of the traversal is described in previous articles [KK89, Ney95b]. Note that we only have to compute intersection between a ray and bilinear patch, which 

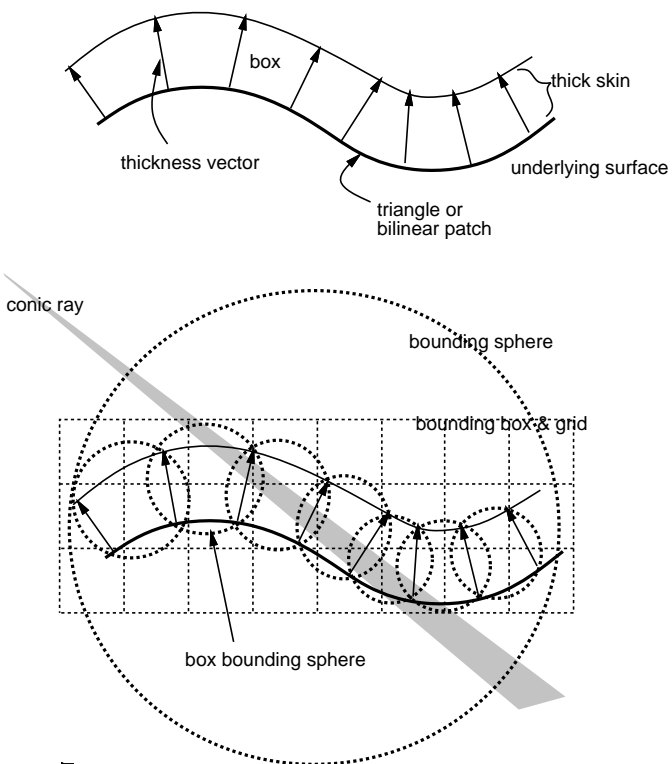

Figure 7: top: thick skin specification. bottom: grid and bounding boxes used for efficient ray-skin intersection computation.

leads to a simple $2 \times 2 Q_{1}$ system $^{3}$ to solve (if the camera is not inside the box).

At this stage we can switch to the $\mathcal{F}$ coordinates which are associated to the skin space. For Kajiya's simple mapping, $\mathcal{T}$ and $\mathcal{F}$ are identical, otherwise we have to cross also this $\mathcal{F}$ space before accessing to a single texel area. This stage (see figure 8 ) does not exist in previous methods. Note that in the skin coordinate system, the ray no longer propagates in a straight line. The trilinear deformation being small, we can either use a simple iterative scheme that gives correct intersections on the $(u, v, w) \operatorname{grid}^{4}$, or approximate the path by a straight line, which is generally sufficient. In the last case, this stage of the traversal is a regular grid crossing, which is easy to implement.

Between two slices of the $(u, v, w)$ grid we are in a texel, so we switch to the reference volume (i.e. the $\mathcal{T}$ space) that we cross linearly. This part of the volume traversal is described in our previous paper [Ney95b], and is similar to classical volumetric rendering. We use a kind of cone tracing to allow adaptative rendering: we know the thickness of the ray at this place, and we estimate the voxel size to be used in the octree regarding this aperture (this is exactly a 3D mipmapping). As described in [Ney95b], another difference compared to volume rendering lies in the fact that the local reflectance is stored in each voxel, and is obtained from the NDF enclosed in that voxel.

\footnotetext{
${ }^{3} \mathrm{~A} Q_{1}$ polynomial is of degree one in each variable, i.e. the highest degree term is $U . V . W$.

${ }^{4}$ The ray direction is expressed in $\mathcal{T}$ space. Assuming it is straight allows to obtain an approximation of the distance to the grid, that we used to follow the path in $\mathcal{S}$ space. We iterate while the point is not close enough to the $(u, v, w)$ grid of $\mathcal{T}$ space, on the wanted slice.
}

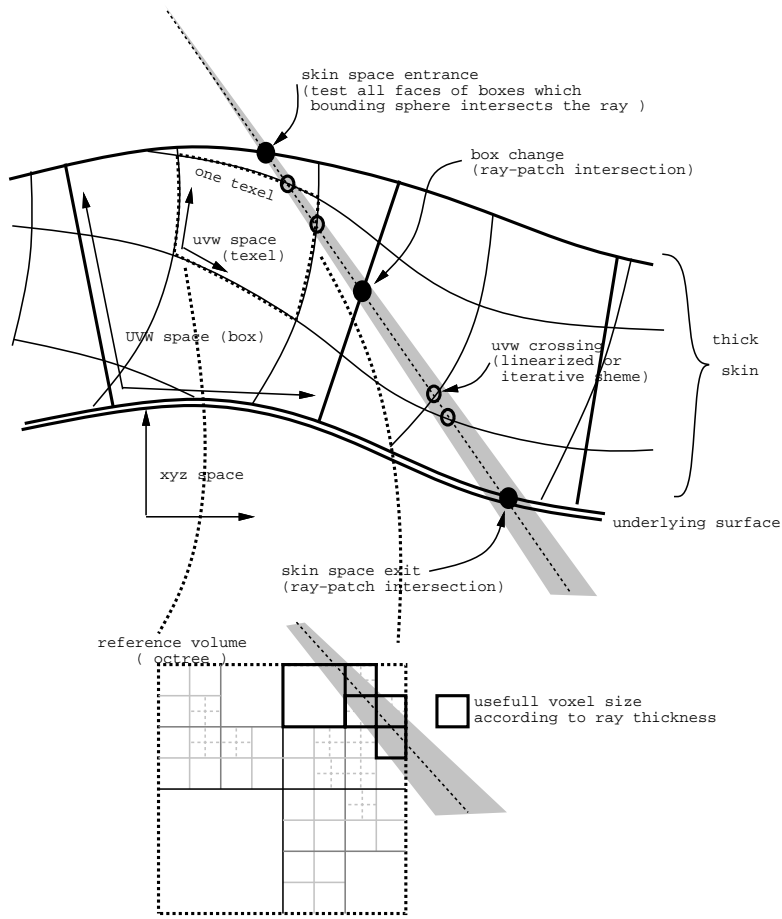

Figure 8: Ray traversal inside the thick skin, crossing the boxes, then the texels, then the voxels at the adapted size.

\subsection{Continuous and discrete jittering}

A simple mapping induces a very regular aspect. This aspect can be improved by jittering the diverse parameters.

When the texture is continuous, the pattern has to be cyclic so that no frontier is visible. Continuous perturbations can be obtained by jittering texture coordinates, texel thickness, or thick skin orientation (by modifying height vectors). A Perlin noise is wellsuited to this purpose.

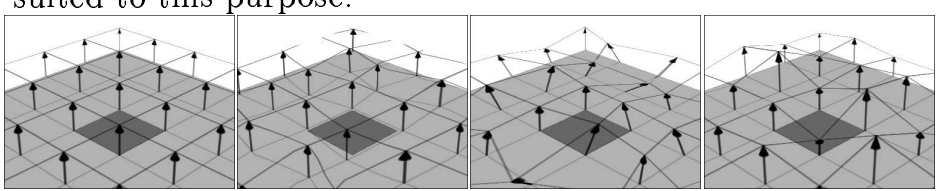

Figure 9: From left to right: simple mapping; jittering of texture coordinates, vectors direction, vectors length.

When the pattern consists of an isolated object, there is no longer a continuity constraint (if the object does not cross the texel edge). Then, more manipulations can be used: alternating reference volume and material, displacing and scaling the texel content, applying symetries or $\pi / 2$ rotations (i.e. either axis permutation).

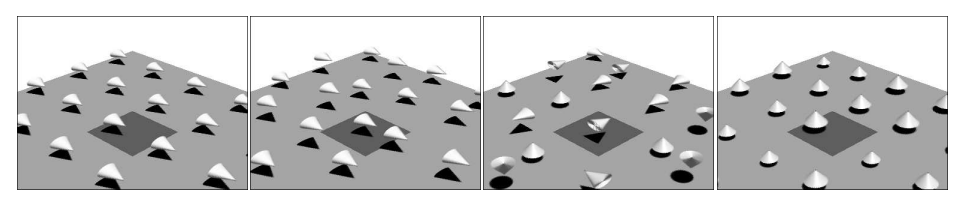

Figure 10: From left to right: simple mapping; perturbation due to displacing, rotating, scaling. 


\section{$5 \quad$ Results}

We have applied our technique to generate verdant landscapes. The computations are done on an SGI Indy having a $200 \mathrm{Mhz}$ R4400 processor. The rendering is achieved with a single ray per pixel, and requires 20 minutes on average.

The first scene is a lawn, covering a hill made of 1400 bilinear patches. The mapping is jittered by modifying the height vectors. Each texel contains 16 grass blades and sometimes a flower. In total 22000 blades and 700 flowers are present. The blades are generated using parabolic trajectories, similar to particle systems, with the section having a ' $\mathrm{V}$ ' shape. A $128^{3}$ resolution is used.

The second scene represents 512 spaced trees on a flat land made of 1024 bilinear patches. The trees are modeled using 6 iterations of a L-system, yielding 2154 branches and 6336 leaves. The reference volume contains one isolated tree. Since the camera is very close to the trees, we have taken a $512^{3}$ volumetric resolution (the volume is compressed more than $99.9 \%$ ). A single model is used, and is modified along the texels by changing the size, the orientation, the position and the material. Note the continuous transition between further and closer trees.

The last example is forest covered mountains, using 25000 trees mapped on a 1404 bilinear patches surface. The texture is continuous so that the reference volume has a cyclic content, consisting of two trees clipped on the edges of the volume. The two trees are generated using the same L-system used for the previous scene, with different parameters. Texture coordinates and height are jittered. The trees are seen from a far point of view most of the time, but the camera sometimes gets closer: a $256^{3}$ resolution is used. Note that the scene contains around 200 million primitives (branches and leaves), reproduces fine shadows, gives smooth transitions while zooming, with very little aliasing, using a single ray per pixel.
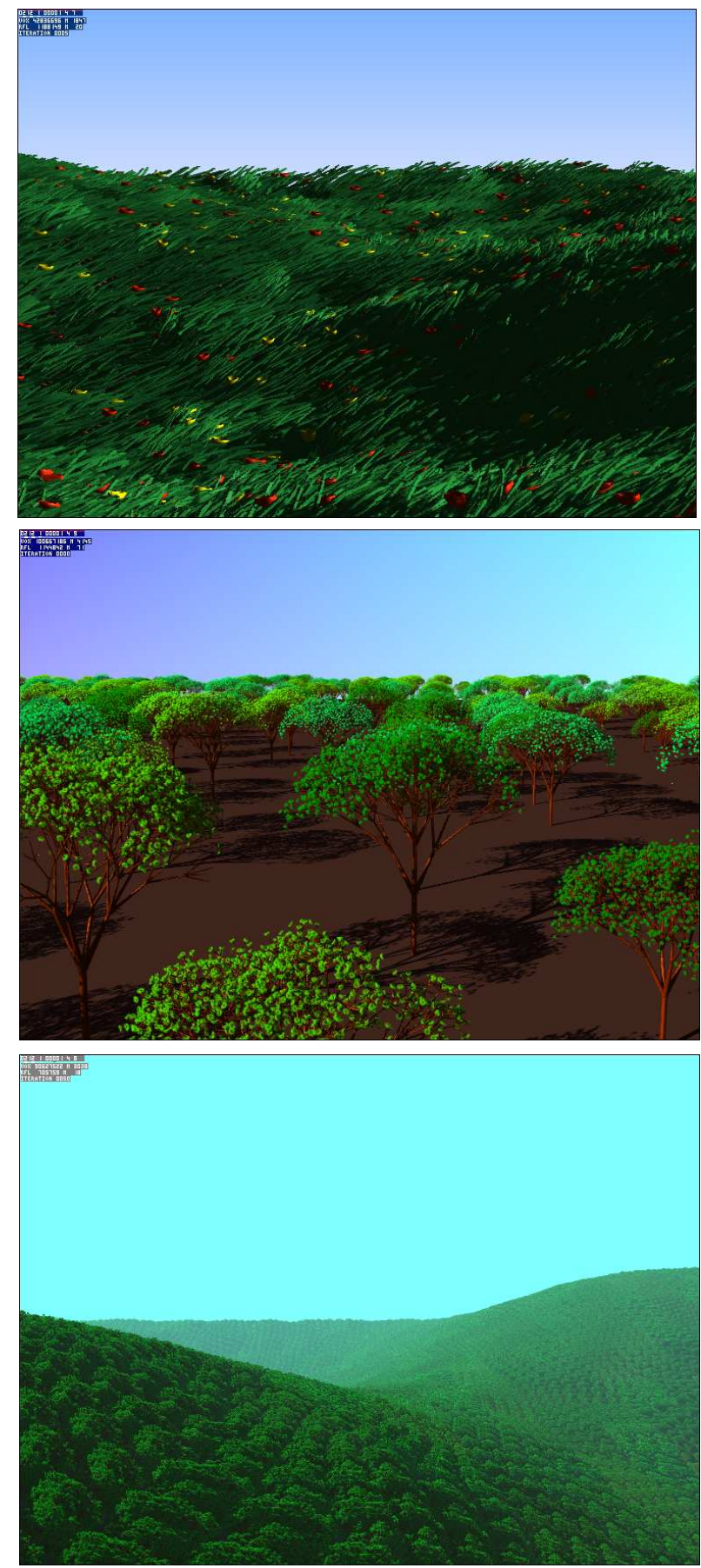


\section{Conclusion}

Two important problems of the volumetric texture representation were texel construction and mapping. We have presented here how to convert various usual representations such as meshes or L-systems into texels and how to define and render mapped texels.

Volumetric texture is now a complete, convenient and efficient tool: a scene is conveniently built and animated using the textural aspect, the pattern can be designed using usual modeling tools, the rendering is done efficiently with low aliasing in ray-tracing, with the cost depending more on visual complexity than on data complexity.

Texels are nevertheless a textural approach, and they cannot model all existing objects. They are well suited for the synthesis of complex repetitive objects, that occur in many scenes such as landscapes.

A lot more can still be brought to the texel world. One can imagine specialized interactive tools to specify or manipulate the reference volume. On the other hand, texels are a new approach to the level-ofdetail problem. Some studies may be conducted in order to use texels outside the scope of textures, as an alternate geometric representation to be used for distant viewpoints.

\section{References}

[dREF ${ }^{+88}$ ] Phillippe de Reffye, Claude Edelin, Jean Françon, Marc Jaeger, and Claude Puech. Plant models faithful to botanical structure and development. In John Dill, editor, Computer Graphics (SIGGRAPH '88 Proceedings), volume 22(4), pages 151-158, August 1988.

[FR86] Alain Fournier and William T. Reeves. A simple model of ocean waves. In David C. Evans and Russell J. Athay, editors, Computer Graphics (SIGGRAPH '86 Proceedings), volume 20, pages 75-84, August 1986.

[FvDFH90] J. D. Foley, A. van Dam, S. K. Feiner, and J. F. Hughes. Computer Graphics: Principles and Practices (2nd Edition). Addison Wesley, 1990.

[KK89] James T. Kajiya and Timothy L. Kay. Rendering fur with three dimensional textures. In Jeffrey Lane, editor, Computer Graphics (SIGGRAPH '89 Proceedings), volume 23(3), pages 271-280, July 1989.

[Ney95a] Fabrice Neyret. Animated texels. In Eurographics Workshop on Animation and Simulation'95, pages 97-103, September 1995.

[Ney95b] Fabrice Neyret. A general and multiscale method for volumetric textures. In Graphics Interface'95 Proceedings, pages 83-91, May 1995.

[Nom95] Tsukasa Noma. Bridging between surface rendering and volume rendering for multi-resolution display. In 6th Eurographics Workshop on Rendering, June 1995.

[Per85] Ken Perlin. An image synthesizer. In B. A. Barsky, editor, Computer Graphics (SIGGRAPH '85 Proceedings), volume 19(3), pages 287-296, July 1985.

[PH89] Ken Perlin and Eric M. Hoffert. Hypertexture. In Jeffrey Lane, editor, Computer Graphics (SIGGRAPH '89 Proceedings), volume 23(3), pages 253262, July 1989.

[PLH88] Przemyslaw Prusinkiewicz, Aristid Lindenmayer, and James Hanan. Developmental models of herbaceous plants for computer imagery purposes. In John Dill, editor, Computer Graphics (SIGGRAPH '88 Proceedings), volume 22, pages 141-150, August 1988.

[RB85] William T. Reeves and Ricki Blau. Approximate and probabilistic algorithms for shading and rendering structured particle systems. In B. A. Barsky, editor, Computer Graphics (SIGGRAPH '85 Proceedings), volume 19(3), pages 313-322, July 1985.

[Ree83] W. T. Reeves. Particle systems - a technique for modeling a class of fuzzy objects. ACM Trans. Graphics, 2:91-108, A pril 1983.

[Sam90] Hanan Samet. Design and Analysis of Spatial Data Structures. Addison-Wesley, Reading, Massachusetts, 1990.

[Shi92] Mikio Shinya. Hierarchical 3D texture. In Graphics Interface '92 Workshop on Local Illumination, pages 61-67, May 1992.

[Smi84] Alvy Ray Smith. Plants, fractals and formal languages. In Hank Christiansen, editor, Computer Graphics (SIGGRAPH '84 Proceedings), volume 18, pages $1-10$, July 1984.

[WP95] Jason Weber and Joseph Penn. Creation and rendering of realistic trees. In Robert Cook, editor Computer Graphics (SIGGRAPH'95 Proceedings), pages 119-128, August 1995. 
Unit ${ }^{\prime}$ de recherche INRIA Lorraine, Technopôle de Nancy-Brabois, Campus scientifique, 615 rue du Jardin Botanique, BP 101, 54600 VILLERS LÈS NANCY

Unit'e de recherche INRIA Rennes, Irisa, Campus universitaire de Beaulieu, 35042 RENNES Cedex

Unit'e de recherche INRIA Rhône-Alpes, 46 avenue F'elix Viallet, 38031 GRENOBLE Cedex 1

Unit'e de recherche INRIA Rocquencourt, Domaine de Voluceau, Rocquencourt, BP 105, 78153 LE CHESNAY Cedex

Unit'e de recherche INRIA Sophia-Antipolis, 2004 route des Lucioles, BP 93, 06902 SOPHIA-ANTIPOLIS Cedex

\section{Éditeur}

INRIA, Domaine de Voluceau, Rocquencourt, BP 105, 78153 LE CHESNAY Cedex (France)

ISSN 0249-6399 